OPEN ACCESS

Edited by:

Stefano Masiero,

University of Padua, Italy

Reviewed by:

Maria Chiara Maccarone, University Hospital of Padua, Italy Jannis Papathanasiou,

Medical University - Sofia, Bulgaria

*Correspondence: Nicola Veronese nicola.veronese@unipa.it

Specialty section: This article was submitted to Endocrinology of Aging, a section of the journal Frontiers in Endocrinology

Received: 29 October 2021 Accepted: 26 November 2021 Published: 16 December 2021

Citation: Veronese N, Stefanac S, Koyanagi A, Al-Daghri NM, Sabico S, Cooper C, Rizzoli R, Reginster J-Y, Barbagallo $M$,

Dominguez LJ, Smith L and

Maggi S (2021) Lower Limb Muscle Strength and Muscle Mass

Are Associated With Incident

Symptomatic Knee Osteoarthritis: A Longitudinal Cohort Study.

Front. Endocrinol. 12:804560. doi: 10.3389/fendo.2021.804560

\section{Lower Limb Muscle Strength and Muscle Mass Are Associated With Incident Symptomatic Knee Osteoarthritis: A Longitudinal Cohort Study}

\author{
Nicola Veronese ${ }^{1,2 *}$, Sinisa Stefanac ${ }^{3}$, Ai Koyanagi ${ }^{4,5}$, Nasser M. Al-Daghri ${ }^{2}$, \\ Shaun Sabico ${ }^{2}$, Cyrus Cooper ${ }^{6,7,8}$, Renè Rizzoli ${ }^{9}$, Jean-Yves Reginster ${ }^{10}$, \\ Mario Barbagallo ${ }^{1}$, Ligia J. Dominguez ${ }^{1}$, Lee Smith ${ }^{11}$ and Stefania Maggi ${ }^{12}$ \\ ${ }^{1}$ Department of Internal Medicine, Geriatric Section, University of Palermo, Palermo, Italy, ${ }^{2}$ Biochemistry Department, \\ College of Science, King Saud University, Riyadh, Saudi Arabia, ${ }^{3}$ Institute of Outcomes Research, Centre for Medical \\ Statistics, Informatics and Intelligent Systems, Medical University of Vienna, Vienna, Austria, ${ }^{4}$ Research and Development \\ Unit, Parc Sanitari Sant Joan de Déu, Universitat de Barcelona, Fundació Sant Joan de Déu, Barcelona, Spain, ${ }^{5}$ Instituto de \\ Salud Carlos III, Centro de Investigación Biomédica en Red de Salud Mental, Centro de Investigación Biomédica en Red de \\ Salud Mental (CIBERSAM), Madrid, Spain, ${ }^{6}$ Oxford National Institute for Health Research (NIHR) Musculoskeletal Biomedical \\ Research Unit, Nuffield Department of Orthopaedics, Rheumatology and Musculoskeletal Sciences, Nuffield Orthopaedic \\ Centre, University of Oxford, Oxford, United Kingdom, 7 Medical Research Council (MRC) Lifecourse Epidemiology Unit, \\ Southampton General Hospital, University of Southampton, Southampton, United Kingdom, ${ }^{8}$ National Institute for Health \\ Research Nutrition Biomedical Research Centre, Southampton General Hospital, University of Southampton and University \\ Hospital Southampton National Health System (NHS) Foundation Trust, Southampton, United Kingdom, ${ }^{9}$ Division of Bone \\ Diseases, Department of Internal Medicine Specialties, Geneva University Hospitals and Faculty of Medicine, Geneva, \\ Switzerland, ${ }^{10}$ Department of Public Health, Epidemiology and Health Economics, CHU Sart Tilman B23, University of Liege, \\ Liège, Belgium, ${ }^{11}$ The Cambridge Centre for Sport and Exercise Sciences, Department of Life Sciences, Anglia Ruskin \\ University, Cambridge, United Kingdom, ${ }^{12}$ National Research Council, Neuroscience Institute, Aging Branch, Padova, Italy
}

Recent literature suggests that sarcopenia, often represented by low lower limbs muscle mass and strength, can be considered a potential risk factor for knee osteoarthritis (OA), but the available literature is still limited. We therefore aimed to investigate whether sarcopenia is associated with a higher risk of radiographic $(R O A)$ and symptomatic knee $\mathrm{OA}(\mathrm{S} \times \mathrm{OA})$ in a large cohort of North American people in the context of the OA initiative. Sarcopenia at baseline was diagnosed in case of low skeletal muscle mass (i.e., lower skeletal mass index) and poor performance in the chair stands test. The outcomes of interest for this study included ROA (radiographical osteoarthritis) if a knee developed a Kellgren and Lawrence $(\mathrm{KL})$ grade $\geq 2$ at follow-up, and SxOA (symptomatic osteoarthritis) defined as new onset of a combination of painful knee OA. Altogether, 2,492 older participants (mean age: 68.4 years, 61.4\% females) were included. At baseline, sarcopenia was present in $6.1 \%$ of the population. No significant difference in ROA prevalence was observed between those with and without sarcopenia $(p=0.76)$, whilst people with sarcopenia reported a significant higher prevalence of SxOA $(p<0.0001)$. Using a logistic regression analysis, adjusting for potential confounders at baseline and the 
diagnosis of sarcopenia during follow-up, sarcopenia was associated with a higher incidence of knee SxOA (odds ratio, OR=2.29; 95\% Cl [confidence interval]: 1.42-3.71; $\mathrm{p}=0.001)$, but not knee ROA (OR=1.48; 95\% Cl: 0.53-4.10; $\mathrm{p}=0.45)$. In conclusion, sarcopenia could be associated with a higher risk of negative knee OA outcomes, in particular symptomatic forms.

Keywords: sarcopenia, osteoarthritis of the knee, older people, epidemiology, muscle mass and function

\section{INTRODUCTION}

Osteoarthritis (OA) is the most prevalent type of arthritis (1) and a very common long-term disabling chronic condition (2) characterized by the deterioration of cartilage in the joints (3). Evidence suggests that $\mathrm{OA}$ is the leading cause of disability worldwide with very high personal, social and economic burdens (4). The prevalence of OA increases with age and is more common in women, people with obesity and those with joint trauma (5). The most prevalent musculoskeletal disease in older adults is knee OA which affects at least $19 \%$ of American adults aged 45 years or older (6). Knee OA is characterized by symptomatic and/or radiographic evidence, such as increased pain, functional/joint instability, as well as increased risk of muscle loss and muscle weakness (3). Deterioration of muscle quality and quantity have been linked to sarcopenia $(7,8)$, thus putting the adults with knee OA at high risk of developing this condition (9).

Based on the latest revised European consensus on definition and diagnosis $(2,10)$, sarcopenia is a generalized and progressive muscle disorder with an increased likelihood of a variety of poor health outcomes such as falls and fractures (11-13), impaired mobility (14) and gradual loss of independence to perform activities of everyday living (15), eventually leading to respiratory and cardiac diseases $(16,17)$, low quality of life (QoL) (18) and premature death (19, 20). Moreover, sarcopenia is now recognized to begin earlier in life and is not merely related to ageing as previously presumed (21).

Even though sarcopenia often accompanies OA (22), the association between them is still unclear and due to the conflicting results and insufficient evidence, no agreement has been reached $(23,24)$. Considering the increasing evidence of negative health outcomes that are associated with these two conditions (25), we aimed to investigate whether sarcopenia is associated with a higher risk of radiographic (ROA) and symptomatic knee OA (SxOA) in a large cohort of North American people followed-up for 4 years.

\section{MATERIALS AND METHODS}

\section{Data Source and Subjects}

Data for this study were obtained from the Osteoarthritis Initiative (OAI) database (https://nda.nih.gov/oai/). In the OAI, participants were recruited across four clinical sites in the United States of America (Baltimore, MD; Pittsburgh, PA;
Pawtucket, RI; and Columbus, OH) between February 2004 and May 2006. In the OAI project, individuals were included if they: (1) had knee OA with knee pain for a 30-day period in the past 12 months or (2) were at high risk of developing knee OA (e.g. obese/overweight, family traits for knee OA) (26). For the aims of this work, the data were collected at baseline, in the screening evaluations and in subsequent evaluations until four years of follow-up.

All participants provided written informed consent. The OAI study was given full ethics approval by the institutional review board of the OAI Coordinating Center, at the University of California in San Francisco.

\section{Sarcopenia Definition (Exposure)}

For the definition of sarcopenia, we used the criteria of the revised European consensus on the definition and diagnosis of sarcopenia (10). Sarcopenia was defined as a chair stand test time $>15$ seconds for 5 repetitions (muscle strength parameter) and low skeletal muscle mass (SMM) as reflected by lower skeletal mass index (SMI) (body composition parameter) (10).

SMM was calculated based on the equation proposed by Lee and colleagues (27): $\mathrm{SMM}=0.244^{\star}$ weight $+7.8^{\star}$ height $+6.6^{\star}$ sex $0.098^{*}$ age + race -3.3 [where female $=0$ and male $=1$; race $=0$ (White and Hispanic), race $=1.9$ (Black) and race $=-1.6$ (Asian)]. SMM was further divided by body mass index (BMI) based on weight and height measured by a trained nurse, to create a SMI (28). Low SMM was defined as the lowest quartile of the SMI based on sex-stratified values (29).

\section{Assessment of Knee OA Outcomes}

At baseline and during follow-up examinations, individuals had full knee assessments which included both clinical and radiographic examinations. A fixed flexion posterior-anterior radiograph, which was read centrally for Kellgren and Lawrence (KL) grade, was made for all the participants. In addition, participants were asked regarding knee pain, the following question: 'During the past 30 days, have you had pain, aching, or stiffness in your right/left knee on most days?'.

The outcomes of interest for this study included: (1) ROA (radiographical osteoarthritis) if a knee developed a KL grade $\geq 2$ at follow-up among those without this condition at baseline and (2) SxOA (symptomatic osteoarthritis), defined as the presence of a combination of painful knee OA. The assessment of the knee OA outcomes was made, other than at baseline, at V01 (12 months), V03 (24 months), V05 (36 months), and V06 (48 months). 


\section{Covariates}

Several covariates at baseline other than age and sex were identified as potential confounding factors based on previous literature (30). These included: race (whites vs. others); educational attainment (college or higher vs. others); yearly income (< vs $\geq \$ 50,000$ or missing data); smoking habits; physical activity evaluated using the total score for the Physical Activity Scale for the Elderly scale (PASE) (31); Charlson Comorbidity Index score (32), a validated general health measure of self-reported comorbidities; the number of medications used; daily energy intake (in Kcal).

\section{Statistical Analyses}

Data on continuous variables were normally distributed according to the Kolmogorov-Smirnov test. Data were consequently presented as means and standard deviation values (SD) for quantitative measures, and percentages for all categorical variables by the presence or absence of sarcopenia at baseline. $\mathrm{P}$ values were calculated using an independent $\mathrm{T}$ test for continuous variables and a chi-square test for categorical parameters.

To assess the association between sarcopenia and the outcomes of interest during follow-up, a logistic regression analysis was applied, since a survival analysis was not possible due to lack of information on the precise date of event. The basic adjusted model included age and gender. The fully adjusted model included all parameters associated with the outcomes of interest ( $p$-value $<0.10$ ) or significantly different between sarcopenic and non-sarcopenic subjects ( $\mathrm{p}$-value $<0.05$ ). Multicollinearity among covariates was assessed through variance inflation factor (VIF) (33), taking a cut-off of 2 as the criterion for exclusion. No covariates were excluded using this criterion. Adjusted odds ratios (OR) and 95\% confidence intervals (CI) were calculated to estimate the strength of the associations between sarcopenia at baseline and incident knee OA outcomes.

A $p<0.05$ was deemed statistically significant. All analyses were performed using SPSS ${ }^{\circledR}$ software version 21.0 for Windows (SPSS Inc., Chicago, Illinois).

\section{RESULTS}

\section{Sample Selection}

The OAI database initially included a total of 4,796 participants. We excluded 2,211 individuals since they were less than 60 years of age, 52 since no data regarding body composition or chair stands time were available, and 41 for not having data regarding race. Therefore, 2,492 participants were included, as shown in Figure 1.

\section{Descriptive Characteristics}

The cohort consisted of 1,529 females $(61.4 \%)$, with a mean age of 68.4 years ( \pm 5.4 years; range: $60-79$ years). The prevalence of sarcopenia at baseline was $6.1 \%$, affecting 153 subjects. At baseline, $58 \%$ were affected by knee ROA and, of them, $24.4 \%$ knee SxOA.
Table 1 shows the baseline characteristics by the presence of sarcopenia. Compared to the 2,239 participants without sarcopenia, sarcopenic subjects were significantly older, more sedentary, with a lower educational level and were poorer (Table 1). Sarcopenic individuals had a greater number of comorbidities, and they used a higher number of medications. Finally, no significant difference in ROA prevalence was observed between sarcopenic and nonsarcopenic individuals $(\mathrm{p}=0.76)$, whilst people with sarcopenia reported a significantly higher prevalence of SxOA (69.4\% vs. 49.5\%; p $<0.0001$ ) than their counterparts (Table 1).

\section{Sarcopenia and Incident Knee Osteoarthritis Outcomes}

During the four years of follow-up, the incidence of ROA was $10.7 \%$ and that of SxOA $46.9 \%$. As shown in Table 2, using a logistic regression analysis, adjusting for potential confounders at baseline and the diagnosis of sarcopenia during follow-up, sarcopenia was associated with a higher incidence of knee SxOA (OR=2.29; 95\%CI: 1.42-3.71; $\mathrm{p}=0.001)$, but not knee ROA (OR=1.48; 95\%CI: 0.53-4.10; $\mathrm{p}=0.45)$.

\section{DISCUSSION}

In this research involving 2,492 older people, we found that the presence of sarcopenia at baseline was significantly associated with a higher risk of symptomatic knee OA, over four years of follow-up.

The overall prevalence of sarcopenia at baseline in our study was just under $10 \%$, which is in accordance to the latest systematic review and meta-analysis of general population studies (34). The existing literature supports our finding of sarcopenia in older people $(35,36)$ as well as those with lower educational level and lower income (37), more comorbidities, higher medication intake and more sedentary lifestyle $(38,39)$.

Other works already explored the potential association between sarcopenia and knee OA outcomes in older people. Recently, Andrews et al. reported that sarcopenia could be associated with a higher risk of sarcopenia, in Health, Aging, and Body Composition participants (40). Interestingly, our findings did not show any significant difference between ROA prevalence and people with or without sarcopenia, according to the paper of Andrews et al. (40). However, a significantly higher prevalence of symptomatic knee OA was reported. In another study, whose aim was to explore the prevalence and characteristics of pain associated with sarcopenia, it was found that the prevalence of pain was much higher in participants with sarcopenia than their counterparts (41). These findings overall suggest that sarcopenia could be associated with higher risk of pain associated to knee $\mathrm{OA}$, indicating the need of early identification of these patients for tailored interventions.

For example, physical exercise interventions could be suggested in people with sarcopenia and without symptomatic knee OA since this kind of intervention is able to prevent further muscle mass loss (42), incident knee OA (in particular forms associated to pain) (43), and pain itself (44). In this regard, it 


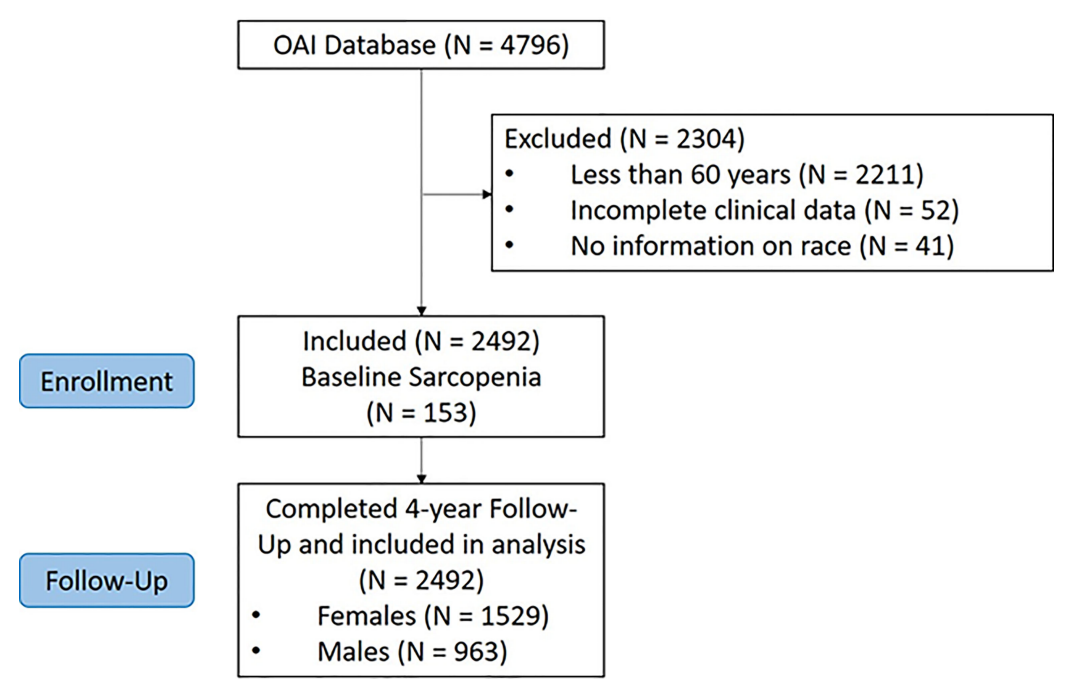

FIGURE 1 | Flow-chart of the participants included.

should be acknowledged that muscle strengthening exercises can improve physical performance and muscle strength parameters in people affected by sarcopenia (45), having the potential to probably prevent symptomatic knee OA.
The strengths of our study are the long duration of follow-up, the several knee OA outcomes assessed, and the large sample size included. However, our findings should be interpreted within some important limitations. First, the participants of the OAI

TABLE 1 | Baseline characteristics of participants according to the presence of sarcopenia.

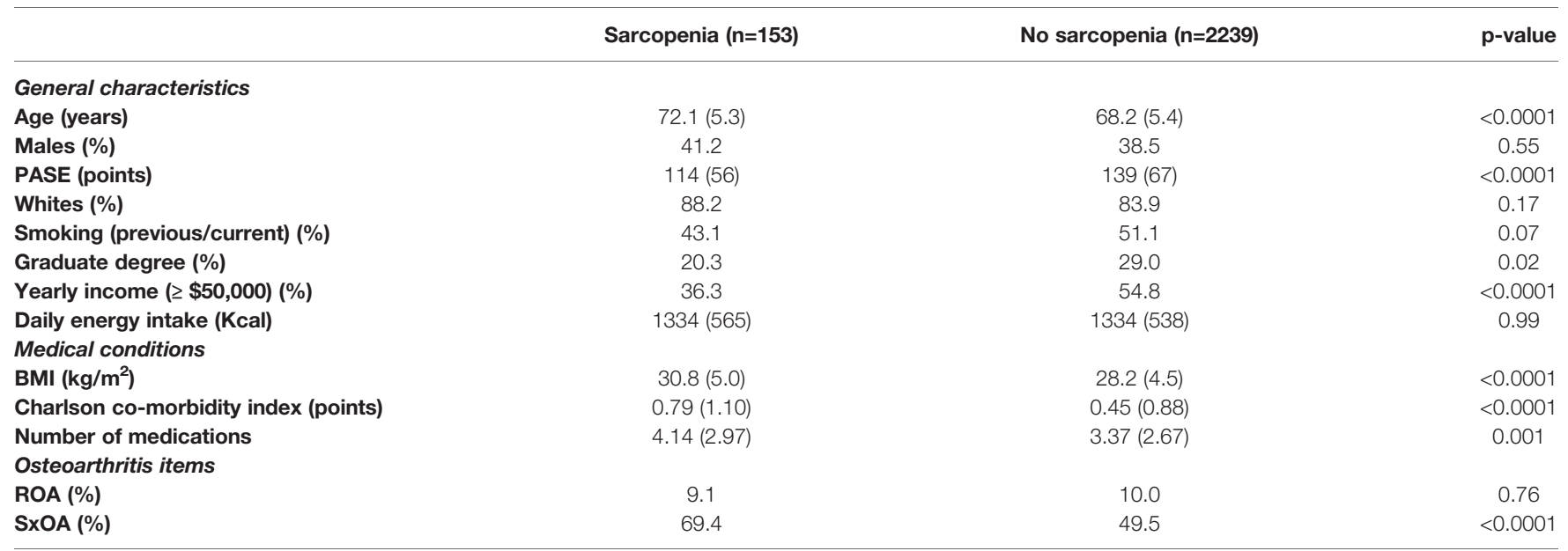

The data are presented as mean (standard deviation) for continuous variables and percentages (\%) for categorical outcomes.

CES-D, Center for Epidemiologic Studies Depression Scale; PASE, Physical Activity Scale for the Elderly; BMI, body mass index; OA, osteoarthritis; ROA, radiographic OA; SxOA, symptomatic knee $O A$.

TABLE 2 | Association between baseline sarcopenia and incident knee OA outcomes.

\begin{tabular}{|c|c|c|c|c|c|}
\hline & $\mathbf{N}$ & Basic adjusted model (OR, 95\% Cl) & p-value & Fully adjusted model ${ }^{1}(\mathrm{OR}, 95 \% \mathrm{Cl})$ & p-value \\
\hline ROA & 847 & $1.38(0.51-3.76)$ & 0.52 & $1.48(0.53-4.10)$ & 0.45 \\
\hline SxOA & 1683 & $2.51(1.56-4.02)$ & $<0.001$ & $2.29(1.42-3.71)$ & 0.001 \\
\hline
\end{tabular}

All the data are presented as odds ratios (95\% confidence intervals).

${ }^{1}$ Basic adjusted model included as covariates age (as continuous) and sex;

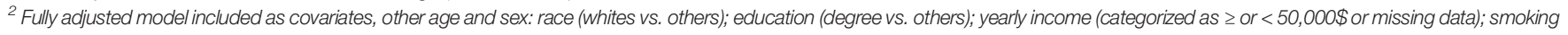
habits (current and previous vs. others); Physical Activity Scale for Elderly score (as continuous); Charlson co-morbidity index; number of medications used; energy intake (as continuous).

$\mathrm{Cl}$, confidence intervals; $\mathrm{OR}$, odds ratio; $R O A$, radiographic $O A$; SxOA, symptomatic knee OA. 
were at high risk or already had knee OA. Thus, our results cannot be extended to the general population. Second, the observational nature of our findings can introduce another bias in our results, although we tried to correct this limitation using analyses adjusted for potential confounders. Finally, body composition was based on a population equation and not on direct assessment. However, this has been validated against gold standard methods such as magnetic resonance imaging and dualenergy X-ray absorptiometry (27). At the same time, sarcopenia was identified using lower limbs performance and muscle mass, whilst handgrip strength is the preferred method for diagnosis sarcopenia (10).

In conclusion, our study suggests that sarcopenia could be associated with a higher risk of negative knee OA outcomes and in particular symptomatic forms. Our findings further suggest the importance of early detection of sarcopenia, in order to implement appropriate preventive treatment against the progression of knee OA.

\section{DATA AVAILABILITY STATEMENT}

The dataset supporting the conclusions of this article is freely available in https://nda.nih.gov/oai/.

\section{ETHICS STATEMENT}

The OAI study was given full ethics approval by the institutional review board of the OAI Coordinating Center, at the University

\section{REFERENCES}

1. Picavet HS, Hazes JM. Prevalence of Self Reported Musculoskeletal Diseases Is High. Ann Rheum Dis (2003) 62:644-50. doi: 10.1136/ard.62.7.644

2. Leyland KM, Gates LS, Sanchez-Santos MT, Nevitt MC, Felson D, Jones G, et al. Knee Osteoarthritis and Time-to All-Cause Mortality in Six Community-Based Cohorts: An International Meta-Analysis of Individual Participant-Level Data. Aging Clin Exp Res (2021) 33:529-45. doi: 10.1007/ s40520-020-01762-2

3. Hunter DJ, Bierma-Zeinstra S. Osteoarthritis. Lancet (2019) 393:1745-59. doi: 10.1016/S0140-6736(19)30417-9

4. Yahaya I, Wright T, Babatunde OO, Corp N, Helliwell T, Dikomitis L, et al. Prevalence of Osteoarthritis in Lower Middle- and Low-Income Countries: A Systematic Review and Meta-Analysis. Rheumatol Int (2021) 41:1221-31. doi: 10.1007/s00296-021-04838-y

5. G.B.D. Disease, I. Injury and C. Prevalence. Global, Regional, and National Incidence, Prevalence, and Years Lived With Disability for 310 Diseases and Injuries, 1990-2015: A Systematic Analysis for the Global Burden of Disease Study 2015. Lancet (2016) 388:1545-602. doi: 10.1016/S0140-6736(16)31678-6

6. Lawrence RC, Felson DT, Helmick CG, Arnold LM, Choi H, Deyo RA, et al. Estimates of the Prevalence of Arthritis and Other Rheumatic Conditions in the United States. Part II Arthritis Rheum (2008) 58:26-35. doi: 10.1002/ art.23176

7. Kim HT, Kim HJ, Ahn HY, Hong YH. An Analysis of Age-Related Loss of Skeletal Muscle Mass and Its Significance on Osteoarthritis in a Korean Population. Korean J Intern Med (2016) 31:585-93. doi: 10.3904/ kjim.2015.156 of California in San Francisco. The patients/participants provided their written informed consent to participate in this study.

\section{AUTHOR CONTRIBUTIONS}

Manuscript preparation, NV, SiS, NA-D, and ShS. Critical revision, SM, LS, CC, RR, and J-YR. Data interpretation, MB, and LD. Statistical analysis, NV. All authors contributed to the article and approved the submitted version.

\section{FUNDING}

The authors thank the support of the Researchers Supporting Project (RSP-2021/21) King Saud University, Riyadh, Saudi Arabia. The OAI is a public-private partnership comprised of five contracts (N01-AR-2-2258, N01-AR-2-2259, N01-AR-22260, N01-AR-2-2261, and N01-AR-2-2262) funded by the National Institutes of Health, a branch of the Department of Health and Human Services, and conducted by the OAI Study Investigators. Private funding partners include Merck Research Laboratories, Novartis Pharmaceuticals Corporation, GlaxoSmithKline, and Pfizer, Inc. Private sector funding for the OAI is managed by the Foundation for the National Institutes of Health. This manuscript was prepared using an OAI public use data set and does not necessarily reflect the opinions or views of the OAI investigators, the $\mathrm{NIH}$, or the private funding partners.
8. Lee DC, Shook RP, Drenowatz C, Blair SN. Physical Activity and Sarcopenic Obesity: Definition, Assessment, Prevalence and Mechanism. Future Sci OA (2016) 2:FSO127. doi: 10.4155/fsoa-2016-0028

9. Shorter E, Sannicandro AJ, Poulet B, Goljanek-Whysall K. Skeletal Muscle Wasting and Its Relationship With Osteoarthritis: A Mini-Review of Mechanisms and Current Interventions. Curr Rheumatol Rep (2019) 21:40. doi: 10.1007/s11926-019-0839-4

10. Cruz-Jentoft AJ, Bahat G, Bauer J, Boirie Y, Bruyere O, Cederholm T, et al. @ the Extended Group for, Sarcopenia: Revised European Consensus on Definition and Diagnosis. Age Ageing (2019) 48:16-31. doi: 10.1093/ageing/ afy 169

11. Bischoff-Ferrari HA, Orav JE, Kanis JA, Rizzoli R, Schlogl M, Staehelin HB, et al. Comparative Performance of Current Definitions of Sarcopenia Against the Prospective Incidence of Falls Among Community-Dwelling Seniors Age 65 and Older. Osteoporos Int (2015) 26:2793-802. doi: 10.1007/s00198-0153194-y

12. Schaap LA, van Schoor NM, Lips P, Visser M. Associations of Sarcopenia Definitions, and Their Components, With the Incidence of Recurrent Falling and Fractures: The Longitudinal Aging Study Amsterdam. J Gerontol A Biol Sci Med Sci (2018) 73:1199-204. doi: 10.1093/gerona/glx245

13. Beaudart C, Bauer JM, Landi F, Bruyère $O$, Reginster J-Y, Hiligsmann M. Experts' Preferences for Sarcopenia Outcomes: A Discrete-Choice Experiment From a Working Group of the European Society for Clinical and Economic Aspects of Osteoporosis, Osteoarthritis and Musculoskeletal Diseases (ESCEO) in Collaboration With the European Union of Geriatric Medicine Society (EUGMS). Aging Clin Exp Res (2021) 33:1079-83. doi: 10.1007/ s40520-021-01794-2 
14. Morley JE, Abbatecola AM, Argiles JM, Baracos V, Bauer J, Bhasin S, et al. Sarcopenia With Limited Mobility: An International Consensus. J Am Med Dir Assoc (2011) 12:403-9. doi: 10.1016/j.jamda.2011.04.014

15. Dos Santos L, Cyrino ES, Antunes M, Santos DA, Sardinha LB. Sarcopenia and Physical Independence in Older Adults: The Independent and Synergic Role of Muscle Mass and Muscle Function. J Cachexia Sarcopenia Muscle (2017) 8:245-50. doi: 10.1002/jcsm.12160

16. Bone AE, Hepgul N, Kon S, Maddocks M. Sarcopenia and Frailty in Chronic Respiratory Disease. Chron Respir Dis (2017) 14:85-99. doi: 10.1177/ 1479972316679664

17. Bahat G, İlhan B. Sarcopenia and the Cardiometabolic Syndrome: A Narrative Review. Eur Geriatric Med (2016) 7:220-23. doi: 10.1016/j.eurger.2015.12.012

18. Beaudart C, Biver E, Reginster JY, Rizzoli R, Rolland Y, Bautmans I, et al. Validation of the SarQoL(R), a Specific Health-Related Quality of Life Questionnaire for Sarcopenia. J Cachexia Sarcopenia Muscle (2017) 8:23844. doi: $10.1002 / \mathrm{jcsm} .12149$

19. De Buyser SL, Petrovic M, Taes YE, Toye KR, Kaufman JM, Lapauw B, et al. Validation of the FNIH Sarcopenia Criteria and SOF Frailty Index as Predictors of Long-Term Mortality in Ambulatory Older Men. Age Ageing (2016) 45:602-8. doi: 10.1093/ageing/afw071

20. Wu TY, Liaw CK, Chen FC, Kuo KL, Chie WC, Yang RS. Sarcopenia Screened With SARC-F Questionnaire Is Associated With Quality of Life and 4-Year Mortality. J Am Med Dir Assoc (2016) 17:1129-35. doi: 10.1016/ j.jamda.2016.07.029

21. Sayer AA, Syddall H, Martin H, Patel H, Baylis D, Cooper C. The Developmental Origins of Sarcopenia. J Nutr Health Aging (2008) 12:42732. doi: 10.1007/BF02982703

22. Bouchouras G, Sofianidis G, Patsika G, Kellis E, Hatzitaki V. Women With Knee Osteoarthritis Increase Knee Muscle Co-Contraction to Perform Stand to Sit. Aging Clin Exp Res (2020) 32:655-62. doi: 10.1007/s40520-019-01245-z

23. Ho KK, Lau LC, Chau WW, Poon Q, Chung KY, Wong RM. End-Stage Knee Osteoarthritis With and Without Sarcopenia and the Effect of Knee Arthroplasty - a Prospective Cohort Study. BMC Geriatr (2021) 21:2. doi: 10.1186/s12877-020-01929-6

24. Jones TL, Esa MS, Li KHC, Krishnan SRG, Elgallab GM, Pearce MS, et al. Osteoporosis, Fracture, Osteoarthritis \& Sarcopenia: A Systematic Review of Circulating microRNA Association. Bone (2021) 152:116068. doi: 10.1016/ j.bone.2021.116068

25. Cieza A, Causey K, Kamenov K, Hanson SW, Chatterji S, Vos T. Global Estimates of the Need for Rehabilitation Based on the Global Burden of Disease Study 2019: A Systematic Analysis for the Global Burden of Disease Study 2019. Lancet (2021) 396:2006-17. doi: 10.1016/S0140-6736(20)32340-0

26. Eby GA, Eby KL. Rapid Recovery From Major Depression Using Magnesium Treatment. Med Hypotheses (2006) 67:362-70. doi: 10.1016/j.mehy.2006.01.047

27. Lee RC, Wang Z, Heo M, Ross R, Janssen I, Heymsfield SB. Total-Body Skeletal Muscle Mass: Development and Cross-Validation of Anthropometric Prediction Models. Am J Clin Nutr (2000) 72:796-803. doi: 10.1093/ajcn/ 72.3.796

28. Studenski SA, Peters KW, Alley DE, Cawthon PM, McLean RR, Harris TB, et al. The FNIH Sarcopenia Project: Rationale, Study Description, Conference Recommendations, and Final Estimates. J Gerontol A Biol Sci Med Sci (2014) 69:547-58. doi: 10.1093/gerona/glu010

29. Tyrovolas S, Koyanagi A, Olaya B, Ayuso-Mateos JL, Miret M, Chatterji S, et al. Factors Associated With Skeletal Muscle Mass, Sarcopenia, and Sarcopenic Obesity in Older Adults: A Multi-Continent Study. J Cachexia Sarcopenia Muscle (2016) 7:312-21. doi: 10.1002/jcsm.12076

30. Allen KD, Golightly YM. Epidemiology of Osteoarthritis: State of the Evidence. Curr Opin Rheumatol (2015) 27:276-83. doi: 10.1097/BOR. 0000000000000161

31. Washburn RA, McAuley E, Katula J, Mihalko SL, Boileau RA. The Physical Activity Scale for the Elderly (PASE): Evidence for Validity. J Clin Epidemiol (1999) 52:643-51. doi: 10.1016/S0895-4356(99)00049-9

32. Katz JN, Chang LC, Sangha O, Fossel AH, Bates DW. Can Comorbidity be Measured by Questionnaire Rather Than Medical Record Review? Med Care (1996) 34:73-84. doi: 10.1097/00005650-199601000-00006

33. Miles J. Tolerance and Variance Inflation Factor. London, UK: Wiley Stats Ref: Statistics Reference Online (2009).
34. Shafiee G, Keshtkar A, Soltani A, Ahadi Z, Larijani B, Heshmat R. Prevalence of Sarcopenia in the World: A Systematic Review and Meta- Analysis of General Population Studies. J Diabetes Metab Disord (2017) 16:21. doi: 10.1186/s40200-017-0302-x

35. Walston JD. Sarcopenia in Older Adults. Curr Opin Rheumatol (2012) 24:623-7. doi: 10.1097/BOR.0b013e328358d59b

36. Dodds RM, Murray JC, Robinson SM, Sayer AA. The Identification of Probable Sarcopenia in Early Old Age Based on the SARC-F Tool and Clinical Suspicion: Findings From the 1946 British Birth Cohort. Eur Geriatr Med (2020) 11:433-41. doi: 10.1007/s41999-020-00310-5

37. Perez-Sousa MA, Pozo-Cruz JD, Cano-Gutierrez CA, Izquierdo M, Ramirez-Velez R. High Prevalence of Probable Sarcopenia in a Representative Sample From Colombia: Implications for Geriatrics in Latin America. J Am Med Dir Assoc (2021) 22:859-64.el. doi: 10.1016/j.jamda.2020.10.021

38. Pacifico J, Geerlings MAJ, Reijnierse EM, Phassouliotis C, Lim WK, Maier AB. Prevalence of Sarcopenia as a Comorbid Disease: A Systematic Review and Meta-Analysis. Exp Gerontol (2020) 131:110801. doi: 10.1016/j.exger. 2019.110801

39. Gong G, Wan W, Zhang X, Liu Y, Liu X, Yin J. Correlation Between the Charlson Comorbidity Index and Skeletal Muscle Mass/Physical Performance in Hospitalized Older People Potentially Suffering From Sarcopenia. BMC Geriatr (2019) 19:367. doi: 10.1186/s12877-019-1395-5

40. Andrews JS, Gold LS, Nevitt M, Heagerty PJ, Cawthon PM. Appendicular Lean Mass, Grip Strength, and the Development of Knee Osteoarthritis and Knee Pain Among Older Adults. ACR Open Rheumatol (2021) 3:566-72. doi: $10.1002 / \mathrm{acr} 2.11302$

41. Maruya K, Fujita H, Arai T, Asahi R, Morita Y, Ishibashi H. Sarcopenia and Lower Limb Pain Are Additively Related to Motor Function and a History of Falls and Fracture in Community-Dwelling Elderly People. Osteoporos Sarcopenia (2019) 5:23-6. doi: 10.1016/j.afos.2019.03.002

42. Escriche-Escuder A, Fuentes-Abolafio IJ, Roldán-Jiménez C, Cuesta-Vargas AI. Effects of Exercise on Muscle Mass, Strength, and Physical Performance in Older Adults With Sarcopenia: A Systematic Review and Meta-Analysis According to the EWGSOP Criteria. Exp Gerontol (2021) 151:111420. doi: 10.1016/j.exger.2021.111420

43. Osthoff A-KR, Juhl CB, Knittle K, Dagfinrud H, Hurkmans E, Braun J, et al. Effects of Exercise and Physical Activity Promotion: MetaAnalysis Informing the 2018 EULAR Recommendations for Physical Activity in People With Rheumatoid Arthritis, Spondyloarthritis and Hip/ Knee Osteoarthritis. RMD Open (2018) 4:e000713. doi: 10.1136/rmdopen2018-000713

44. Geneen LJ, Moore RA, Clarke C, Martin D, Colvin LA, Smith BH. Physical Activity and Exercise for Chronic Pain in Adults: An Overview of Cochrane Reviews. Cochrane Database Syst Rev (2017) 141(1):CD011279. doi: 10.1002/ 14651858.CD011279.pub2

45. Choi M, Kim H, Bae J. Does the Combination of Resistance Training and A Nutritional Intervention Have A Synergic Effect on Muscle Mass, Strength, and Physical Function in Older Adults? A Syst Rev Meta-Analysis (2021). doi: 10.21203/rs.3.rs-596096/v1

Conflict of Interest: The authors declare that the research was conducted in the absence of any commercial or financial relationships that could be construed as a potential conflict of interest.

Publisher's Note: All claims expressed in this article are solely those of the authors and do not necessarily represent those of their affiliated organizations, or those of the publisher, the editors and the reviewers. Any product that may be evaluated in this article, or claim that may be made by its manufacturer, is not guaranteed or endorsed by the publisher.

Copyright $\odot 2021$ Veronese, Stefanac, Koyanagi, Al-Daghri, Sabico, Cooper, Rizzoli, Reginster, Barbagallo, Dominguez, Smith and Maggi. This is an open-access article distributed under the terms of the Creative Commons Attribution License (CC BY). The use, distribution or reproduction in other forums is permitted, provided the original author(s) and the copyright owner(s) are credited and that the original publication in this journal is cited, in accordance with accepted academic practice. No use, distribution or reproduction is permitted which does not comply with these terms. 